
.

\title{
Gram-Scale Synthesis and Supramolecular Complex of Precursors of Clar's Hydrocarbon Triangulene
}

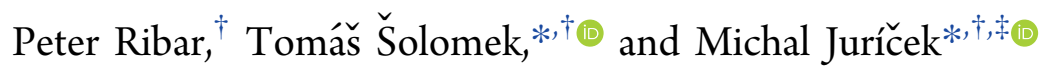 \\ ${ }^{\dagger}$ Department of Chemistry, University of Basel, St. Johanns-Ring 19, CH-4056 Basel, Switzerland \\ ${ }^{\star}$ Department of Chemistry, University of Zurich, Winterthurerstrasse 190, CH-8057 Zurich, Switzerland
}

Supporting Information

ABSTRACT: We present to date the most efficient gramscale synthesis of triangulene-4,8-dione and 12-hydroxytriangulene-4,8-dione, the precursors of Clar's hydrocarbon, in overall yields $>50 \%$. The direct dihydroprecursors of triangulene, obtained upon reduction of triangulene-4,8dione, were stabilized in a supramolecular complex with a

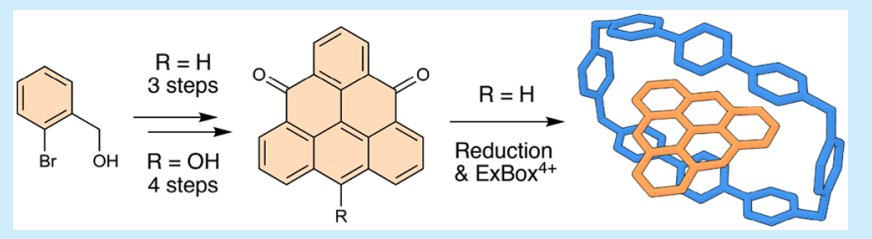
tetracationic cyclophane ExBox ${ }^{4+}$ and characterized by singlecrystal X-ray crystallography. This result represents the first step in an endeavor to stabilize the fragile core of triangulene in an inclusion complex in solution and solid state.

$\mathrm{T}^{\mathrm{k}}$ he "odd" nature of triangulene has provoked the minds of chemists ever since the proposal of Eric Clar in 1950s. ${ }^{1}$ On account of high reactivity, the synthesis of this compound known as Clar's hydrocarbon was not successful until 2001, when Kubo et al. confirmed for the first time the triplet ground state of its trisubstituted derivative by EPR spectroscopy in solution. ${ }^{2}$ After 10 years of (EPR) silence, the chemistry of triangular graphene fragments started to flourish again, and this year, triangulene is featured in the logo of the Symposium on Novel Aromatic Compounds (ISNA) held in Sapporo, Japan. The most recent breakthrough is the imaging of the naked triangulene (2017) and its $\pi$-extended homologues (2019) on various surfaces by using STM/AFM techniques (Figure 1$){ }^{3}$

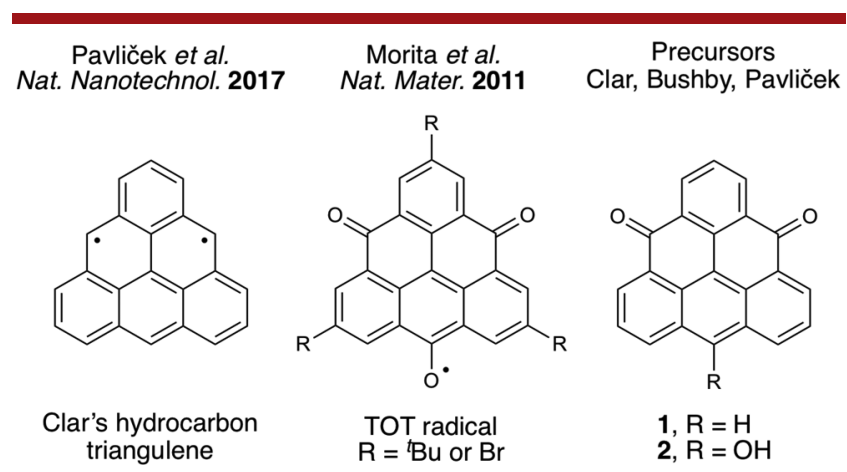

Figure 1. Recent breakthroughs in the chemistry of triangulene.

The first attempts to synthesize triangulene were carried out by Clar. His efforts using the old-days aromatic hydrocarbon chemistry gave rise to two oxo-functionalized precursors $\mathbf{1}$ and 2 (Figure 1) accessible in eight and seven steps with $29 \%$ and $36 \%$ yields, respectively (Scheme 1). ${ }^{4}$ Despite the incredible synthetic methodology advancements in the past 60 years, only two shorter pathways have been reported since then by
Scheme 1. Previous Strategies to Triangulene Precursors Clar, 1951-1954

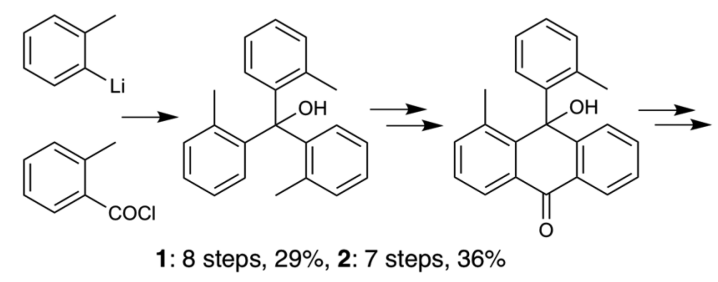

Bushby, 1993-1995

Pavliček, 2017

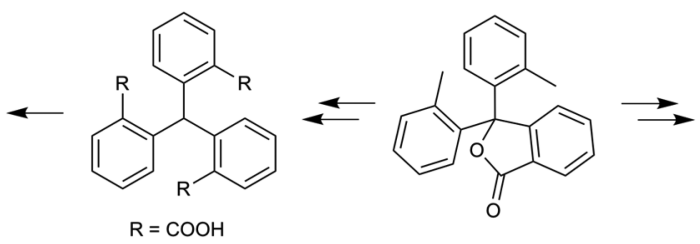

2: 4 steps, $17 \%$

1: 4 steps, $6 \%, 2: 3$ steps, N/A

Bushby $^{5}$ et al. (1995) and Pavliček ${ }^{3 a}$ et al. (2017). None of them, however, surpassed the synthesis of Clar in terms of overall chemical yields.

The oxo-substitution of the triangulene core endows the neutral trioxotriangulene (TOT) radical derived from 2 with chemical stability. This stabilization allowed the exploration of the rich redox properties of TOTs, ${ }^{6}$ which can store up to four electrons reversibly, and the use of this system as a molecular component of cathode-active materials in organic batteries. The tri-tert-butyl TOT exhibited a high discharge capacity of more than $300 \mathrm{~A} \mathrm{~h} \mathrm{~kg}^{-1}$, exceeding those delivered by Li-ion

Received: July 30, 2019

Published: August 15, 2019 
batteries. The unpaired electron in TOT radicals is responsible for the formation of one-dimensional assemblies in the solid state on account of weak $\pi$-stacking. Such assemblies show semiconducting properties with relatively high conductivities of $\sim 10^{-3} \mathrm{~S} \mathrm{~cm}^{-1}$. Mixing of a sample of TOT radical with a sample of its reduced form results in a purely organic mixedvalence salt, exhibiting conductivity that can reach up to $125 \mathrm{~S}$ $\mathrm{cm}^{-1}$. It has also been demonstrated that other TOT derivatives can act as promising metal-free electrocatalysts in the oxygen-reduction reaction. We showed that the tri-tertbutyl derivative of $\mathbf{2}$ can easily be converted into donoracceptor dyads with rare layered solid-state packing motifs. ${ }^{7}$ Finally, because of the extra stabilization provided by the peripheral oxygen atoms, the doubly reduced conjugate base of 2 was the first TOT derivative with a triplet ground state detected by EPR spectroscopy. ${ }^{8}$

Considering the importance of these achievements, both fundamentally and from the standpoint of applications, there is a need for a high-yielding few-step synthesis of $\mathbf{1}$ and $\mathbf{2}$ that can be performed on a gram scale. The common feature of all previously published synthetic strategies (Scheme 1 and Schemes S1-S3) is the preparation of 3,3-di-o-tolylisobenzofuran-1 $(3 H)$-one. This precursor represents the point in synthesis where the approaches of Bushby and Pavliček shorten the original sequence of Clar, although they significantly forfeit the yield. The subsequent oxidation of the two methyl groups in this precursor to carboxylic acids is required to assemble the triangulenedione core in a final acidcatalyzed cyclization. Each strategy uses rather harsh ( $\mathrm{KMnO}_{4} /$ reflux; Bushby, Pavliček) or even dangerous $\left(\mathrm{HNO}_{3} / 200{ }^{\circ} \mathrm{C}\right.$; Clar; precursor on the right in Scheme 1) oxidation conditions. In fact, when we were reproducing the synthetic sequence of Clar, we encountered an explosion during this step in our attempt to oxidize the methyl groups using a pressure flask (Figure S1). A similar dangerous outcome was previously reported in the literature by Bushby et al. ${ }^{9}$ For this reason, we strongly discourage even skilled synthetic organic chemists to use this method.

To avoid these issues, we designed a new synthesis of $\mathbf{1}$ and 2 (Scheme 2), which employs an intermediate that is accessible in a single high-yielding step and which can be oxidized using a milder oxidant. This intermediate, a $C_{3}$-symmetric tetraalcohol 4 , could be successfully obtained in a very good $74 \%$ yield through (1) a lithium-halogen exchange in commercially available 2-bromobenzylalcohol with two equiv of $n-\mathrm{BuLi}$, followed by (2) a 3-fold nucleophilic addition of the resulting aryl lithium salt to diethylcarbonate. This reaction can be easily upscaled to $\sim 50 \mathrm{~g}$ of the starting 2-bromobenzylalcohol without decreasing the yield. Compound 4 does not require purification by column chromatography and can be obtained pure by simple recrystallization from ethyl acetate.

The structure of $\mathbf{4}$ allows for using a milder oxidant to convert the three hydroxymethyl groups into the corresponding carboxylic acids. Our initial attempts with Jones oxidation provided a mixture of $\mathbf{5}$ and $\mathbf{6}$. While the latter is a byproduct that cannot be oxidized to the desired compound 7 , the former possesses a formyl group in place of the expected carboxylic acid. This unexpected result was, however, a fortunate reaction outcome because 5 can be converted directly to $\mathbf{1}$, a reduced form of $\mathbf{2}$ without the hydroxyl group in position 12. The optimized conditions of the Jones oxidation provided 5 in $77 \%$ ( $3.7 \mathrm{~g}$ ) and 6 in $21 \%$ yield. This oxidation is the only step in our sequence that requires purification by column chromatog-

Scheme 2. Synthesis of Triangulene Precursors 1, 2, and $3^{a}$
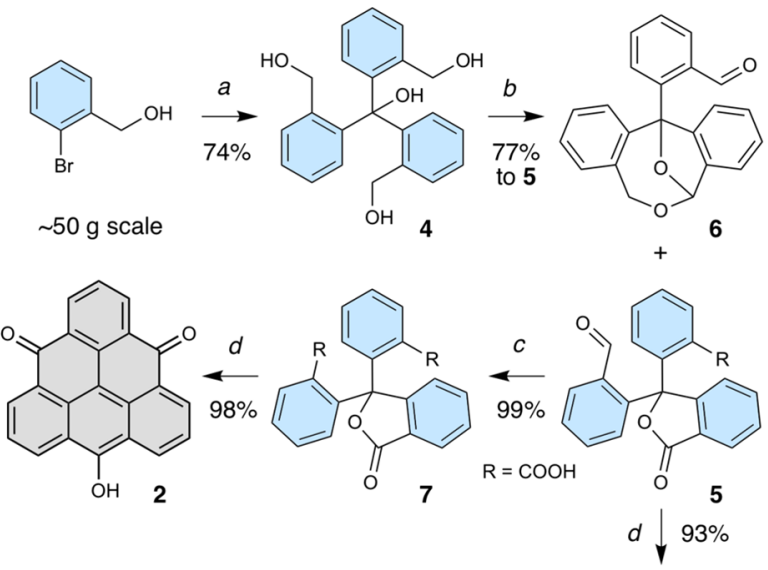

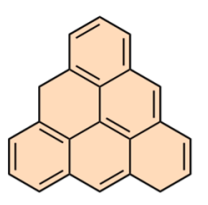

3a

$(10: 1)$

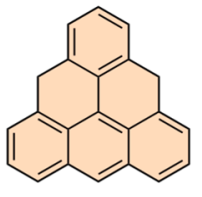

3b

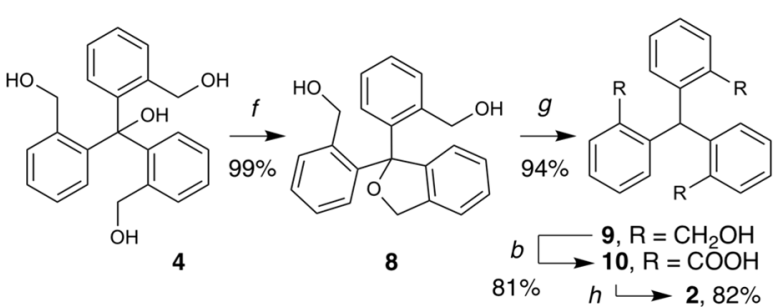

${ }^{a}$ Reaction conditions: (a, 1) $n$-BuLi, THF, $-78{ }^{\circ} \mathrm{C}, 2 \mathrm{~h} ;(\mathrm{a}, 2)$ (EtO) ${ }_{2} \mathrm{CO}, \mathrm{rt}, 7$ days, 74\%; (b) $\mathrm{CrO}_{3}, \mathrm{H}_{2} \mathrm{SO}_{4}$, acetone, reflux, $12 \mathrm{~h}$, 77\% (5), 21\% (6), 81\% (10); (c) $\mathrm{H}_{2} \mathrm{O}_{2}, \mathrm{NaH}_{2} \mathrm{PO}_{4}, \mathrm{HCl}, \mathrm{NaClO}_{2}$, $\mathrm{MeCN}, 10{ }^{\circ} \mathrm{C}, 6 \mathrm{~h}, 99 \%$; (d) Cu, $\mathrm{H}_{2} \mathrm{SO}_{4}, 120{ }^{\circ} \mathrm{C}, 93 \%$ (1), 98\% (2); (e) $\mathrm{LiAlH}_{4}, \mathrm{AlCl}_{3}, \mathrm{Et}_{2} \mathrm{O} / \mathrm{THF}, 3.5 \mathrm{~h}, 11 \%$ (3 cycles); (f) $\mathrm{Me}_{2} \mathrm{SiCl}_{2}$, MeCN, rt, 2 h, 99\%; (g) Li, $\mathrm{C}_{10} \mathrm{H}_{10}$, THF, rt, 14 h, 94\%; (h) $\mathrm{H}_{2} \mathrm{SO}_{4}$, $120{ }^{\circ} \mathrm{C}, 2 \mathrm{~h}, 82 \%$.

raphy. Subsequent Friedel-Crafts acylation of 5, employing concentrated sulfuric acid as the solvent and copper as a reducing agent, ${ }^{4}$ resulted in the formation of 1 in excellent $93 \%$ yield $(1.9 \mathrm{~g})$. This three-step procedure with an overall $53 \%$ yield is the shortest and best-yielding synthesis of $\mathbf{1}$ to date.

Similar cyclization to form $\mathbf{2}$ requires that the formyl group in $\mathbf{5}$ is first oxidized to the carboxylic acid. This step was performed with a $\mathrm{NaClO}_{2} / \mathrm{H}_{2} \mathrm{O}_{2}$ mixture, ${ }^{10}$ yielding 7 in a nearly quantitative yield $(99 \%, 3.1 \mathrm{~g})$ without the need for purification. Finally, cyclization using $\mathrm{Cu} / \mathrm{H}_{2} \mathrm{SO}_{4}$ afforded 2, which was obtained pure upon filtration also almost quantitatively $(98 \%, 2.6 \mathrm{~g})$. This four-step synthetic route gives 2 in an overall $55 \%$ yield, which is $\sim 1.5$ times higher than that of the best available seven-step synthesis of Clar. ${ }^{4}$ Because of low solubility, the structures of both $\mathbf{1}$ and $\mathbf{2}$ were confirmed by $1 \mathrm{D} / 2 \mathrm{D}$ NMR spectroscopy in $\mathrm{D}_{2} \mathrm{SO}_{4}$, including the full assignment of ${ }^{1} \mathrm{H}$ and ${ }^{13} \mathrm{C}$ resonances reported here for the first time (see the Supporting Information).

Since our attempts to prevent the formation of $\mathbf{6}$ in the Jones oxidation of the key tetraalcohol 4 were not successful, we explored an alternative path (Scheme 1, bottom). The key was to avoid the formation of the acetal moiety in 6 by reducing the central hydroxy group in 4 . First, ether intermediate 8 was synthesized upon treatment of $\mathbf{4}$ with 
dimethylsilyl dichloride ${ }^{11}$ in almost a quantitative yield. Then, 8 was reduced by lithium naphthalenide ${ }^{12}$ in $94 \%$ yield to triol 9. Subsequent Jones oxidation provided the tricarboxylic acid 10 in a considerably better yield (81\%) than the method of Bushby et al. (54\%), which required oxidation of two methyl groups by potassium permanganate to form 10 . The final 3fold Friedel-Crafts acylation afforded 2 in $82 \%$ yield. Although this alternative route added an extra synthetic step, its overall yield of $46 \%$ is superior to any of the previously reported syntheses.

To obtain the direct precursor of pristine triangulene, ketotriangulenes $\mathbf{1}$ or $\mathbf{2}$ must be further reduced. Reduction of $\mathbf{1}$ to a mixture of $\mathbf{3 a}$ and $\mathbf{3 b}$ was reported previously twice. ${ }^{3 \mathrm{a}, 13}$ We felt, however, that both the method and the available spectroscopic data to characterize 3 were not sufficiently described, or the samples of 3 were produced in an unsatisfactory purity because of the sensitivity of 3 to oxygen. Our initial attempts to reduce 1 using original conditions $\mathrm{AlCl}_{3} / \mathrm{LiAlH}_{4}$ revealed that this protocol leads only to small quantities of the desired product. It appeared to us that the reason is the low solubility of $\mathbf{1}$. As the reaction progressed, the surface of undissolved solid 1 would be covered by aluminum oxide, a reaction byproduct, which suppressed the reduction to proceed further. To partially overcome this problem, 1 was added into the mixture of $\mathrm{AlCl}_{3} / \mathrm{LiAlH}_{4}$ in small portions. The next important part of the protocol was to isolate soluble red byproducts, presumably various hydroxyl triangulenes formed upon contact with oxygen, during the column chromatography. Even though the presence of oxygen was minimized during the purification steps, formation of these byproducts could not be avoided. They were subjected again to the same reduction conditions, which afforded additional amounts of the desired product. After three cycles, the combined product samples were purified once again by oxygen-free column chromatography, to afford a pure sample of 3 in $11 \%$ overall yield. Although this yield is lower than the reported ones, our method is reliable for obtaining very pure material. The NMR spectroscopy revealed that 3 is formed as a 10:1 mixture of two inseparable dihydro isomers $\mathbf{3 a}$ and $\mathbf{3 b}$. The experimental ratio of $10: 1$ is in an excellent agreement with the populations of $89 \%$ and $11 \%$ for $3 a$ and $3 \mathbf{b}$, respectively, calculated from our DFT data. Both compounds were fully characterized by $2 \mathrm{D}$ NMR spectroscopy, including the full assignment of ${ }^{1} \mathrm{H}$ and ${ }^{13} \mathrm{C}$ resonances, for the first time (see the Supporting Information).

It was reported by Kubo et al. that three tert-butyl groups in the apexes of triangulene improved its kinetic stability and permitted recording an EPR spectrum in a frozen toluene matrix at $123 \mathrm{~K}^{2}$ An intermediate increase of the temperature resulted, however, in a rapid oligo-/polymerization of the diradical in the sample. A better steric protection of triangulene would require introduction of bulky substituents at positions 4 , 8 , and 12 , which is not a straightforward synthetic task because of the unexpected chemical reactivity of $\pi$-extended aromatic ketones such as 1 and $2 .{ }^{14}$ An alternative strategy to prevent bimolecular reactions of triangulene in solution is an inclusion of the fragile triangulene core into a large protective molecular shell or, in other words, stabilizing triangulene in the form of a supramolecular host-guest complex. Such an approach has previously enabled kinetically stabilizing a number of wellknown reactive intermediates. ${ }^{15}$ The first step in this endeavor is a successful formation of a supramolecular complex of triangulene precursors 3 . We selected a tetracationic cyclo- phane $\mathrm{ExBox}^{4+}$ as a promising candidate. ${ }^{16} \mathrm{ExBox}^{4+}$ is known to scavenge electron-rich polyaromatic hydrocarbons from their solutions on account of a favorable donor-acceptor interaction in the formed host-guest complexes, the strength of which correlates with the number of $\pi$-electrons. Dihydroprecursors 3 possess $20 \pi$-electrons each and are expected to form a stable host-guest complex with $\mathrm{ExBox}^{4+}$. Indeed, formation of the $3 \subset \mathrm{ExBox} \cdot 4 \mathrm{PF}_{6}$ complex was confirmed by ${ }^{1} \mathrm{H}$ NMR spectroscopy in solution (Figure 2) and X-ray crystallography in the solid state (Figure 3 ). ${ }^{17}$

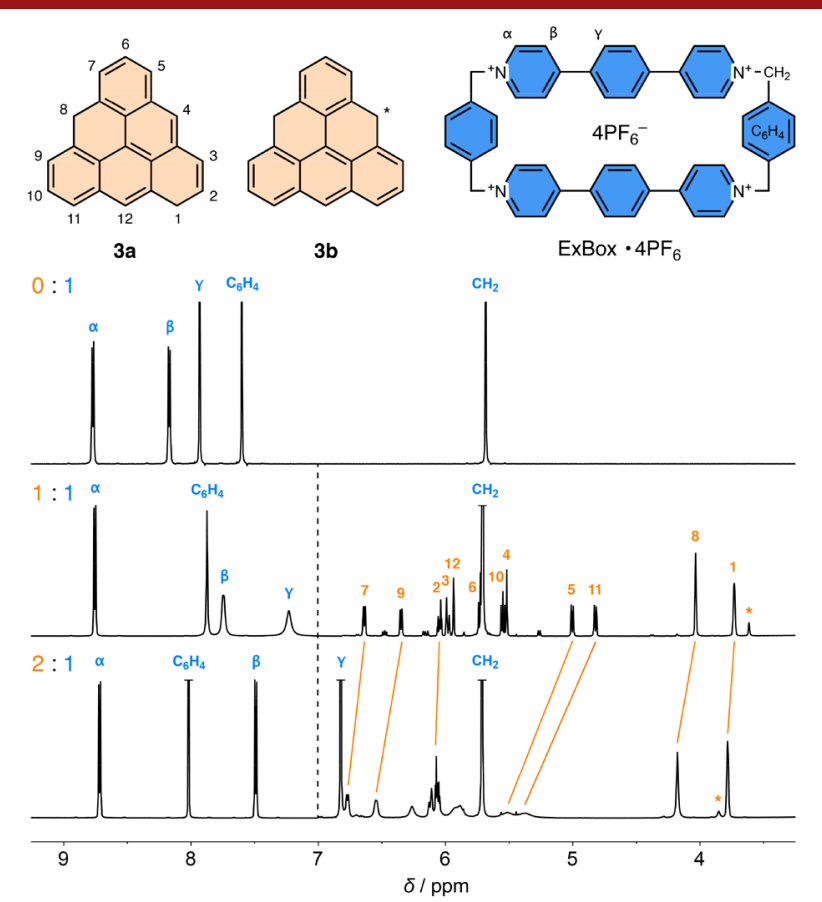

Figure 2. Structures of $3 \mathbf{a}, 3 \mathbf{b}$, and ExBox$\cdot 4 \mathrm{PF}_{6}$ (top) and ${ }^{1} \mathrm{H} \mathrm{NMR}$ spectra $\left(\mathrm{CD}_{3} \mathrm{CN}, 25^{\circ} \mathrm{C}\right.$; bottom $)$ of ExBox$\cdot 4 \mathrm{PF}_{6}$ and its $1: 1$ and $1: 2$ complexes with $3(3 \mathbf{a} / \mathbf{3} \mathbf{b} \sim 10: 1)$. Intensity of the parts of spectra on the right from the dashed line was increased with respect to the left part for clarity.

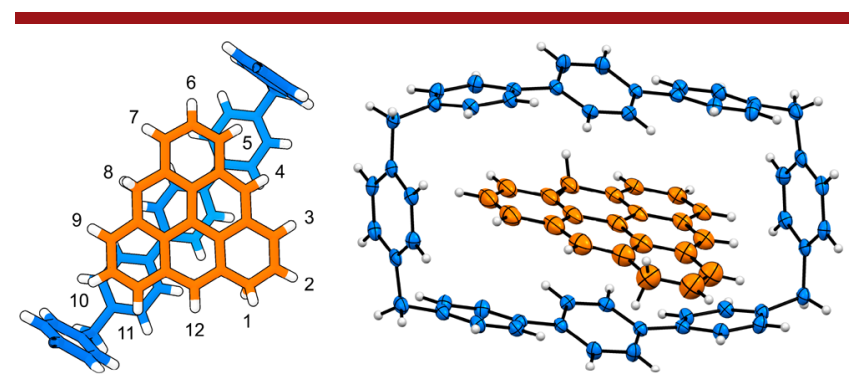

Figure 3. Different crystallographic views of the solid-state structure of $3 C \mathrm{ExBox} \cdot 4 \mathrm{PF}_{6} .{ }^{17}$ The top part of $\mathrm{ExBox}^{4+}$ in the structure on the left is not displayed for clarity. Thermal ellipsoids are shown at a 50\% probability level.

In the ${ }^{1} \mathrm{H}$ NMR spectrum of a 1:1 mixture of 3 and ExBox. $4 \mathrm{PF}_{6}$ in $\mathrm{CD}_{3} \mathrm{CN}$, upfield shifts of $\beta$ and $\gamma$ protons and a downfield shift of the $\mathrm{C}_{6} \mathrm{H}_{4}$ protons for ExBox $4 \mathrm{PF}_{6}$ as well as upfield shifts for all signals of $\mathbf{3 a}$ and $\mathbf{3 b}$ were observed. These shifts are in agreement with those observed for inclusion of aromatic guests inside $\mathrm{ExBox}^{4+}$. X-ray diffraction (XRD) analysis of the single crystal of $3 \subset \mathrm{ExBox} \cdot 4 \mathrm{PF}_{6}$, obtained by a slow vapor diffusion of diisopropyl ether into the acetonitrile 
solution of 3 and $\mathrm{ExBox} \cdot 4 \mathrm{PF}_{6}$, confirmed that 3 resides inside the cavity of $\mathrm{ExBox}^{4+}$. A comparison of the carbon-carbon bond lengths of $\mathbf{3 a}$ (the major species) in the complex with those obtained from DFT calculations suggests that the prevalent of the three possible orientations of $3 \mathbf{a}$ inside the cavity is the one shown in Figure 3 (the longest $\mathrm{C}_{\mathrm{sp} 3}-\mathrm{C}_{\mathrm{sp} 2}$ bonds are in the range 1.45-1.47 $\AA$ by XRD and 1.50-1.52 $\AA$ by DFT). This orientation agrees well with the prevalent orientation in solution: the largest upfield shift is observed for protons 5 and 11, smaller shift for protons 7, 8, and 9, and only a very small shift for protons 1 and 2 (Figure 2, orange lines).

The association constant for the binding of 3 inside ExBox ${ }^{4+}$ elucidated by NMR titration (Figure S3) is $\sim 0.8 \times 10^{4} \mathrm{M}^{-1}$, in between those of 18 - $\pi$-electron [4] helicene $\left(\sim 0.6 \times 10^{4} \mathrm{M}^{-1}\right)$ and 20 - $\pi$-electron perylene $\left(\sim 9 \times 10^{4} \mathrm{M}^{-1}\right) \cdot{ }^{16}$ Although the affinity of 3 toward $\mathrm{ExBox}^{4+}$ is relatively high, so is the rate constant for the guest-exchange process, judging from the presence of only one set of averaged signals for the bound and unbound species (both 3 and $\mathrm{ExBox}^{4+}$ ) in the NMR spectrum at room temperature. As a result, our initial attempts to generate triangulene trapped in the supramolecular complex in solution at room temperature using oxidants such as chloranil or non-nucleophilic bases $\left(\mathrm{NaH},{ }^{t} \mathrm{BuOK}\right)$ were unsuccessful. Increasing the activation energy preventing triangulene from escaping from its inclusion complex is thus inevitable. A pursuit of such a goal is in progress in our laboratories.

In conclusion, we presented the most efficient synthesis to date of triangulene-4,8-dione and 12-hydroxytriangulene-4,8dione. Both compounds were synthesized on gram scales in overall yields $>50 \%$ using a common route with only a single purification by column chromatography. Our simple approach promises a rapid access to $C_{3}$-symmetrically oxo-substituted triangulenes. We also proposed a new strategy to stabilize triangulene in a solution or solid state within a supramolecular complex. By means of NMR spectroscopy and single-crystal Xray diffraction, we demonstrated the first step of this strategy, namely, the complexation of ExBox.4PF 6 and the direct precursor of triangulene, the synthesis of which was here optimized to produce samples of high purity.

\section{ASSOCIATED CONTENT}

\section{S Supporting Information}

The Supporting Information is available free of charge on the ACS Publications website at DOI: 10.1021/acs.orglett.9b02683.

Synthetic procedures and characterization data for all new compounds (PDF)

\section{Accession Codes}

CCDC 1943899 contains the supplementary crystallographic data for this paper. These data can be obtained free of charge via www.ccdc.cam.ac.uk/data_request/cif, or by emailing data_request@ccdc.cam.ac.uk, or by contacting The Cambridge Crystallographic Data Centre, 12 Union Road, Cambridge CB2 1EZ, UK; fax: +44 1223336033.

\section{AUTHOR INFORMATION}

\section{Corresponding Authors}

*E-mail: tomas.solomek@unibas.ch.

*E-mail: michal.juricek@chem.uzh.ch.

ORCID $\odot$

Tomás Solomek: 0000-0003-0013-4116
Michal Jurícek: 0000-0001-5595-431X

Notes

The authors declare no competing financial interest.

\section{ACKNOWLEDGMENTS}

This project received funding from the European Research Council (ERC) under the European Union's Horizon 2020 research and innovation programme (Grant Agreement 716139 ), the Swiss National Science Foundation (SNSF, T.Š./PZ00P2_174175, M.J./PZ00P2_148043, PP00P2_170534), and Experientia Foundation (T.S.). We would liketo thank our colleagues at the University of Basel, namely, PD Daniel Häussinger (2D NMR), Dr. Markus Neuburger and Dr. Alessandro Prescimone (XRD), and Prof. Marcel Mayor for supporting our research. We also acknowledge Leoš Valenta (University of Zurich) for help with NMR measurements.

\section{DEDICATION}

Dedicated to Miroslav Kozák on the occasion of his 60th birthday.

\section{REFERENCES}

(1) (a) Clar, E. Aromatische Kohlenwasserstoffe; Springer-Verlag: Berlin, Germany, 1941; p 311. (b) Clar, E. Aromatische Kohlenwasserstoffe, 2nd ed.; Springer-Verlag: Berlin, Germany, 1952; pp 93-97 and 461-465.

(2) Inoue, J.; Fukui, K.; Kubo, T.; Nakazawa, S.; Sato, K.; Shiomi, D.; Morita, Y.; Yamamoto, K.; Takui, T.; Nakasuji, K. J. Am. Chem. Soc. 2001, 123, 12702-12703.

(3) (a) Pavliček, N.; Mistry, A.; Majzik, Y.; Moll, N.; Meyer, G.; Fox, D. J.; Gross, L. Nat. Nanotechnol. 2017, 12, 308-312. (b) Mishra, S.; Beyer, D.; Eimre, K.; Liu, J.; Berger, R.; Gröning, O.; Pignedoli, C. A.; Müllen, K.; Fasel, R.; Feng, X.; Ruffieux, P. J. Am. Chem. Soc. 2019, 141, 10621-10625. (c) Su, J.; Telychko, M.; Hu, P.; Macam, G.; Mutombo, P.; Zhang, H.; Bao, Y.; Cheng, F.; Huang, Z.-Q.; Qiu, Z.; Tan, S. J. R.; Lin, H.; Jelínek, P.; Chuang, F.-C.; Wu, J.; Lu, J. Sci. Adv. 2019, 5, No. eaav7717.

(4) (a) Clar, E.; Stewart, D. G. J. Am. Chem. Soc. 1953, 75, 26672672. (b) Clar, E.; Stewart, D. G. J. Am. Chem. Soc. 1954, 76, 35043507.

(5) Allinson, G.; Bushby, R. J.; Paillaud, J.-L.; Thornton-Pett, M. J. Chem. Soc., Perkin Trans. 1 1995, 385-390.

(6) (a) Morita, Y.; Nishida, S.; Murata, T.; Moriguchi, M.; Ueda, A.; Satoh, M.; Arifuku, K.; Sato, K.; Takui, T. Nat. Mater. 2011, 10, 947951. (b) Morita, Y.; Murata, T.; Ueda, A.; Yamada, C.; Kanzaki, Y.; Shiomi, D.; Sato, K.; Takui, T. Bull. Chem. Soc. Jpn. 2018, 91, 922931. (c) Ikabata, Y.; Wang, Q.; Yoshikawa, T.; Ueda, A.; Murata, T.; Kariyazono, K.; Moriguchi, M.; Okamoto, H.; Morita, Y.; Nakai, H. npj Quantum Mater. 2017, 2, 27. (d) Murata, T.; Yamada, C.; Furukawa, K.; Morita, Y. Commun. Chem. 2018, 1, 47. (e) Murata, T.; Kotsuki, K.; Murayama, H.; Tsuji, R.; Morita, Y. Commun. Chem. 2019, 2, 46

(7) Ribar, P.; Šolomek, T.; Le Pleux, L.; Häussinger, D.; Prescimone, A.; Neuburger, M.; Jurícek, M. Synthesis 2017, 49, 899-909.

(8) Allinson, G.; Bushby, R. J.; Paillaud, J.-L.; Oduwole, D.; Sales, K. J. Am. Chem. Soc. 1993, 115, 2062-2064.

(9) Allinson, G.; Bushby, R. J.; Jesudason, M. V.; Paillaud, J.-L.; Taylor, N. J. Chem. Soc., Perkin Trans. 2 1997, 147-156.

(10) Dalcanale, E.; Montanari, F. J. Org. Chem. 1986, 51, 567-569.

(11) Wiggins, J. M. Synth. Commun. 1988, 18, 741-749.

(12) Azzena, U.; Demartis, S.; Melloni, G. J. Org. Chem. 1996, 61, 4913-4919.

(13) Hara, O.; Tanaka, K.; Yamamoto, K.; Nakazawa, T.; Murata, I. Tetrahedron Lett. 1977, 18, 2435-2436. 
(14) (a) Ribar, P. Doctoral dissertation, University of Basel, 2019. (b) Li, Y.; Huang, K.-W.; Webster, R. D.; Zeng, Z.; Zeng, W.; Chi, C.; Furukawa, K.; Wu, J. Chem. Sci. 2014, 5, 1908-1914.

(15) See, for example: Cram, D. J.; Tanner, M. E.; Thomas, R. Angew. Chem., Int. Ed. Engl. 1991, 30, 1024-1027.

(16) (a) Barnes, J. C.; Juríček, M.; Strutt, N. L.; Frasconi, M.; Sampath, S.; Giesener, M. A.; McGrier, P. L.; Burns, L. C.; Stern, C. L.; Sarjeant, A. A.; Stoddart, J. F. J. Am. Chem. Soc. 2013, 135, 183192. (b) Barnes, J. C.; Jurícek, M.; Vermeulen, N. A.; Dale, E. J.; Stoddart, J. F. J. Org. Chem. 2013, 78, 11962-11969. (c) Dale, E. J.; Vermeulen, N. A.; Juríček, M.; Barnes, J. C.; Young, R. M.; Wasielewski, M. R.; Stoddart, J. F. Acc. Chem. Res. 2016, 49, 262-273.

(17) Crystal parameters for $3 \subset \mathrm{ExBox}_{4} 4 \mathrm{PF}_{6}:\left[\mathrm{C}_{22} \mathrm{H}_{14} \subset \mathrm{C}_{48} \mathrm{H}_{40} \mathrm{~N}_{4}\right.$. $\left.4\left(\mathrm{PF}_{6}\right) \cdot 2.8\left(\mathrm{C}_{2} \mathrm{H}_{3} \mathrm{~N}\right)\right]$; orange plate, $0.16 \times 0.14 \times 0.03 \mathrm{~mm}$; triclinic, $P \overline{1}$ (no. 2); $a=10.1937(7), b=10.6981(8)$, and $c=18.2104(15) \AA ; \alpha$ $=96.700(5), \beta=106.062(3)$, and $\gamma=107.208(3)^{\circ} ; V=1779.80(14)$ $\AA^{3} ; Z=1 ; T=123 \mathrm{~K} ; \rho_{\text {calc }}=1.536 \mathrm{~g} \mathrm{~cm}^{-3} ; \mu=1.994 \mathrm{~mm}^{-1} ; S=$ 1.1042. CCDC no. 1943899. Molecules of 3 show rotational displacement disorder inside the cavity of $\mathrm{ExBox}^{4+}$ (Figure S2). 